\title{
Addressing the P2P Bootstrap Problem for Small Overlay Networks
}

\author{
David Isaac Wolinsky, Pierre St. Juste, P. Oscar Boykin, and Renato Figueiredo \\ Advanced Computing Information Systems Lab \\ University of Florida
}

\begin{abstract}
Peer-to-Peer (P2P) overlays provide a framework for building distributed applications consisting of few to many resources with features including self-configuration, scalability, and resilience to node failures. Such systems have been successfully adopted in large-scale Internet services for content delivery networks, file sharing, and data storage. In small-scale systems, they can be useful to address privacy concerns as well as support for network applications that lack dedicated servers. The bootstrap problem, finding an existing peer in the overlay, remains a challenge to enabling these services for small-scale P2P systems. In large networks, the solution to the bootstrap problem has been the use of dedicated services, though creating and maintaining these systems requires expertise and resources, which constrain their usefulness and make them unappealing for small-scale systems.

This paper surveys and summarizes requirements that allow peers potentially constrained by network connectivity to bootstrap small-scale overlays through the use of existing public overlays. In order to support bootstrapping, a public overlay must support the following requirements: a method for reflection in order to obtain publicly reachable addresses, so peers behind network address translators and firewalls can receive incoming connection requests; communication relaying to share public addresses and communicate when direct communication is not feasible; and rendezvous for discovering remote peers, when the overlay lacks stable membership. After presenting a survey of various public overlays, we identify two overlays that match the requirements: XMPP overlays, such as Google Talk and Live Journal Talk, and Brunet, a structured overlay based upon Symphony. We present qualitative experiences with prototypes that demonstrate the ability to bootstrap small-scale private structured overlays from public Brunet or XMPP infrastructures.
\end{abstract}

\section{INTRODUCTION}

While P2P overlays provide a scalable, resilient, and selfconfiguring platform for distributed applications, their adoption rate for use across the Internet has been slow outside of large-scale systems, such as data distribution and communication. General use of decentralized, P2P applications targeting homes and small/medium businesses (SMBs) has been limited in large part due to difficulty in decentralized discovery of P2P systems - the bootstrap problem — further inhibited by constrained network conditions due to firewalls and NATs (network address translators). While these environments could benefit from P2P, many of these users lack the resources or expertise necessary to bootstrap private ${ }^{1} \mathrm{P} 2 \mathrm{P}$ overlays particularly when the membership is unsteady and across wide-area network environments where a significant amount of

\footnotetext{
${ }^{1}$ In the context of this paper, private implies that the overlay's purpose is not for general use. Once established, such overlays can support privacy in communication; however, overlay security is beyond the scope of this paper.
}

(or all) peers may be unable to initiate direct communication with each other due to firewalls and NATs.

Examples of large-scale P2P systems include Skype, BitTorrent, and Gnutella. Skype is a voice over P2P system, whereas BitTorrent and Gnutella are used for file sharing. The bootstrapping in these systems typically relies on overlay maintainers using high availability systems for bootstrapping, bundling their connection information with the application for distribution. When the application is started, it uses these high availability servers to connect with other peers in the system. Alternatively, some services constantly crawl the network and place peer lists on dedicated web sites. A new peer wishing to join the network queries the web site and then attempts to connect to the peers on that list.

In smaller-scale systems, P2P interests focus on decentralization. For example, users may desire to run an application at many distributed sites, but the application lacks dedicated central servers to provide discovery or rendezvous service for peers. In contrast, dedicated, centralized $\mathrm{P} 2 \mathrm{P}$ service providers, such as LogMeIn's Hamachi, a P2P VPN, may collect usage data, which the users may wish to remain private, or are not free for use.

Many applications make sense for small-scale overlay usage, including multiplayer games, especially those that lack dedicated online services; private data sharing; and distributed file systems. Clearly, a small P2P system could be bootstrapped by one or more users of the system running on public addresses, distributing addresses out-of-band, instructing their peers to add that address to their $\mathrm{P} 2 \mathrm{P}$ application, and then initiate bootstrapping; but these types of situations are an exception and not the norm. Ultimately, the users would be enhanced significantly through approaches that can make decentralized bootstrapping transparent through minimal and intuitive interaction with the $\mathrm{P} 2 \mathrm{P}$ component.

The basic bootstrapping process can be broken down into two components: finding a remote peer, and connecting to it and then successively more peers. When a node begins, it contacts various bootstrap servers, until it successfully connects with one, upon which they exchange information. The bootstrap server may inquire into the overlay for the best set of peers for the new peer and respond with that information or it may respond with its existing neighbor set. At which point, the peer attempts to connect with those peers. This process continues aggressively until the peer arrives at a steady state, either connecting with a specific set of or a 


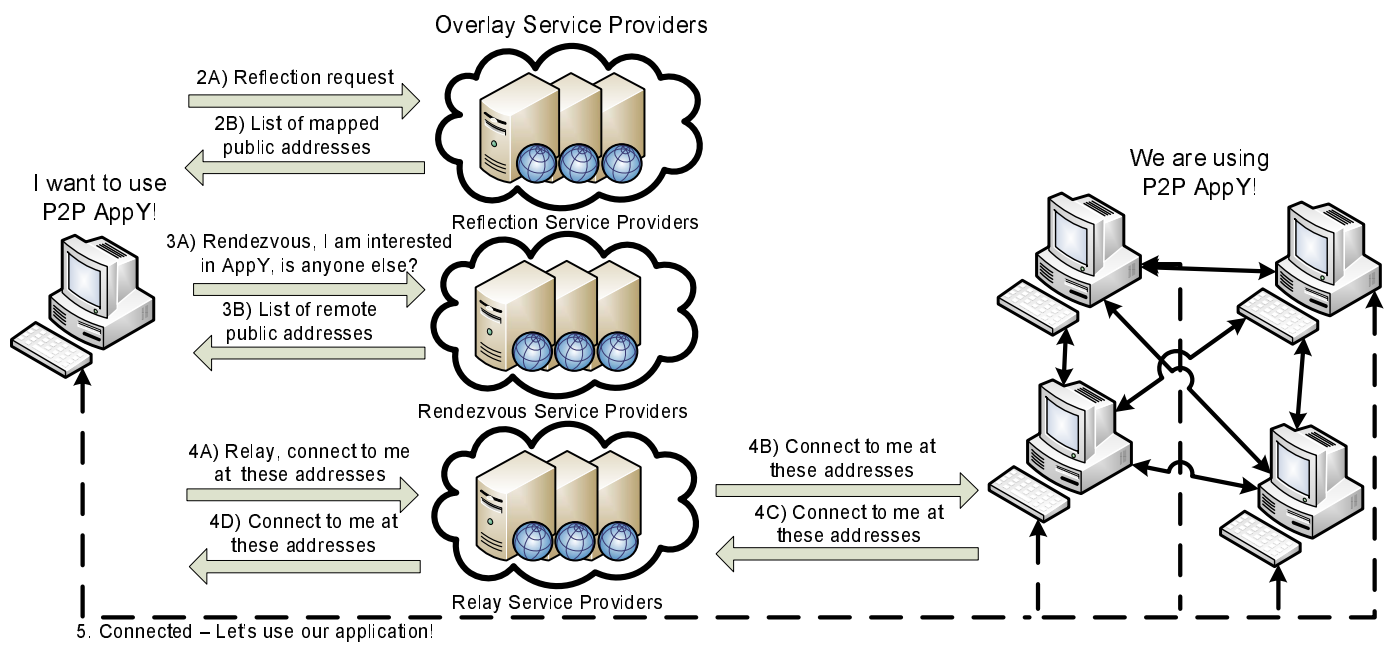

Fig. 1. Bootstrapping a P2P system using an existing (generic) overlay.

number of peers. Afterwards, the P2P logic becomes passive, only reacting to churn from new incoming or outgoing peers.

Overlay support for constrained peers, i.e., those behind NATs and restrictive firewalls, requires additional features to support all-to-all connectivity for peers in the overlay. The instantiation of P2P systems for private use could become overly burdensome, potentially relying on significant human interaction to bootstrap them, for example, by relaying connection information through phone calls and e-mail. Even if this is feasible, this sort of interaction is undesirable; P2P systems should be self-discovering so that users need to do minimal amount of work to take advantage of them and adhoc systems stress this point. In addition, these may rely on centralized components; if they become unavailable, which is a possibility since most users lack the expertise in configuring highly available systems, the system will not be accessible.

To address this, we explore the use of existing public overlays as a means to bootstrap private overlays. There are many existing public overlays with high availability, such as Skype, Gnutella, XMPP (Extensible Messaging and Presence Protocol), and BitTorrent; by leveraging these systems, system integrators can easily enable users to seamlessly bootstrap their own private $\mathrm{P} 2 \mathrm{P}$ systems. In the preceding paragraphs, we identified the components necessary for bootstrapping a homogeneous system; now we expand them for environments to support the bootstrapping of a private overlay from a public overlay with consideration for network constrained peers. The public overlay must support the following mechanisms as illustrated in Figure 1:

1) Reflection - A method for obtaining global application and IP addresses or identifier for a peer that can be shared with others to enable direct communication.

2) Relaying - A method for peers to exchange arbitrary data, when a direct IP link is unavailable.

3) Rendezvous - A method for identifying peers interested in the same P2P service.

This work motivates from the belief that while small-scale P2P systems are attractive for decentralized systems, the overheads relating to creating and maintaining bootstrap services make them unfeasible. A public overlay can be used to transparently bootstrap a private overlay with minimal user interaction.

The requirements are presented and verified in the context of two prototype implementations: a XMPP (Jabber) [1] and Brunet [2]. XMPP-based overlays are commonly used as chat portals, such as GoogleTalk and Facebook Chat. XMPP also supports an overlay amongst servers forming through the XMPP Federation, which allows inter-domain communication amongst chat peers, so that users from various XMPP servers can communicate with each other. Brunet provides generic $\mathrm{P} 2 \mathrm{P}$ abstractions as well as an implementation of the Symphony structured overlay. We present the architecture for these systems, the lessons learned in constructing and evaluating them, and provide an analysis of the latency to establish peer connectivity in a small-scale private Brunet overlay with NATconstrained nodes.

The organization of this paper follows. Section II overviews common P2P overlay technologies, motivating examples for this work, existing solutions to the bootstrapping problem, and NAT challenges in P2P systems. In Section III, we present a survey of overlays, applying the requirements for private overlay bootstrapping to them, and then show in detail how they can be applied to Brunet and XMPP. Our implementation is described in Section IV. In Section V, we then perform a timing evaluation of bootstrapping overlays using our prototype PlanetLab and discuss experiences in deploying the system. Finally, we conclude the paper with Section VI.

\section{MOTIVATION AND BACKGROUND}

The most well-known Internet P2P systems consist of a very large number of nodes and users who benefit from the sheer scale of the system to accomplish tasks such as sharing large files. There are also several applications that can benefit from techniques developed for P2P systems to deliver features that are desirable in small-scale systems, providing self-organizing frameworks upon which applications can be built to support resource aggregation and collaboration. An example is found in virtual private networks (VPNs), which provide end-to-end virtual network connectivity among trusted peers. 
Private overlays enable truly decentralized, P2P VPNs. A P2P VPN enables the reuse of existing network applications on a P2P overlay without modifications. The challenges of reflection, relaying, and rendezvous exist significantly in these systems. In centralized VPNs like OpenVPN, a dedicated server provides for all three services. P2P VPN solutions (such as Hamachi) provide similar VPN functionality, with the improvement that peers can form direct connections with each other which bypass the server. A decentralized P2P VPN solution like SocialVPN [3] relies on a dedicated bootstrap overlay that runs on PlanetLab. Using the techniques described in this paper, SocialVPN could be extended so that it can be bootstrapped into private systems without additional user configuration and relying on sustainable large-scale public overlays.

Large-scale decentralized $\mathrm{P} 2 \mathrm{P}$ architectures that can assist with the bootstrap problem are unstructured and structured systems. Unstructured systems [4], [5] are generally constructed by peers attempting to maintain a certain amount of connections to other peers in the $\mathrm{P} 2 \mathrm{P}$ system, whereas structured systems organize into well-defined topologies, such as trees, 1-D rings, or hypercubes. Though unstructured systems are typically simpler to bootstrap and maintain, they rely on global knowledge, flooding, or stochastic techniques to search for information in an overlay, creating potential scalability constraints. Alternatively, structured systems [6], [7], [8], [9], [10] have guaranteed search time typically with a lower bound of $O(\log N)$ and in some cases even $O(1)$ [11]. The most common feature found in structured overlays is the support for a decentralized storage / retrieval system called a distributed hash table (DHT), that maps keys with associated data to specific node IDs in an overlay.

Another subset found of $\mathrm{P} 2 \mathrm{P}$ systems are those that are not fully decentralized, and deal with the bootstrap problem explicitly through centralized resources. These include "P2P VPNs" like Hamachi, earlier file-sharing systems like the original Napster, and tracker-based BitTorrent. BitTorrent differentiates itself by using the trackers as a gateway into the overlay; once inside, peers exchange connection information with each other directly, relegating the tracker as a fall back. This approach has enabled BitTorrent to be modified to support trackerless torrents through using a DHT.

\section{A. Current Bootstrap Solutions}

As described in the introduction, the simple case of bootstrapping is limited to one peer attempting to find an active peer in the overlay in order for itself to become a member. The large-scale providers have resources not readily available to small-scale overlays. This section reviews existing techniques and those being developed and describes their application to small-scale systems.

When using dedicated bootstrap overlays, a service provider hosts one or more bootstrap resources. Peers desiring to join the overlay query bootstrap nodes, until a successful connection is made to one. The bootstrap server will then assist in connecting the peer to other nodes in the P2P system.
Bootstrap nodes are either packaged with the application at distribution time or through a meta data file, such as in BitTorrent. Drawbacks to this approach for small, ad-hoc pools include that the same server would have to be used every time to bootstrap the system, or users would have to reconfigure their software to connect to new bootstrap servers over time; at least one peer must have a publicly accessible address; and a bootstrap server can become a single point of failure.

Another commonly used approach for large-scale systems is the use of a host cache [12]. Clients post current connection information to dedicated web services, a host cache, that in turn communicate with other host caches. For small, ad-hoc networks, a host cache acts no differently than a centralized rendezvous point, requiring that at least one peer has a publicly accessible address.

"P2P VPN's" [13] use of a BitTorrent tracker is similar to the host cache concept. The tracker hosts file meta data and peers involved in sharing. For the VPN, the peer registers a virtual file used to organize the peers, a form of rendezvous. Each peer in the VPN queries the tracker regarding the file, registers its IP address, and receives other active "sharers" IP addresses. Peers on public addresses or using UPNP are able to receive incoming connections from all other peers. The problem with this approach is that it is heavily user-driven. A user must register with each BitTorrent tracker individually and maintain a connection with each of them, in order to handle cases where BitTorrent trackers go offline. In addition, this does not use the BitTorrent trackers in a normal fashion, so it may be banned by tracker hosts.

Research has shown that peers can use the locality properties of recent IP addresses in a large-scale P2P system to make intelligent guesses about other peers in the $\mathrm{P} 2 \mathrm{P}$ system using an approach called random probing [14], [15]. The results show that, in a network of tens to hundreds of thousands of peers, a bootstrapping peer can find an active peer in 100 guesses to 2,000 guesses, depending on the overlay. The approach does not really apply well to small-scale systems, especially when peers are constrained by NATs and firewalls.

Rather than distribute an IP address, which points explicitly to some location in the Internet, a small P2P network can apply a name abstraction around one peer in the overlay using Dynamic DNS [16]. Peers share a DNS entry, which points to a bootstrap server. When the peers detect that the bootstrap server is offline, at random time intervals they will update the DNS entry with their own. The application of this approach is well-suited to small, ad-hoc groups, as the service could be distributed across multiple Dynamic DNS registrations. However, sharing a DNS entry requires trusting all peers in the overlay, making it easy for malicious peers to inhibit system bootstrapping. Also the approach requires that at least one peer be publicly addressable; if a non-publicly addressable peer updates the cache inadvertently, it could delay or permanently prevent peers from creating a $\mathrm{P} 2 \mathrm{P}$ system. The results reported in [16] were simulation-based and did not determine how well a dynamic DNS handles rapid changing of name to IP mappings. 


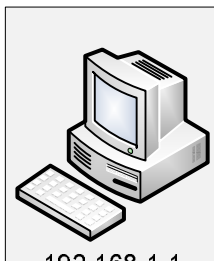

192.168.1.1

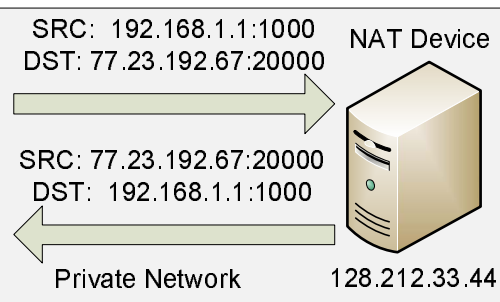

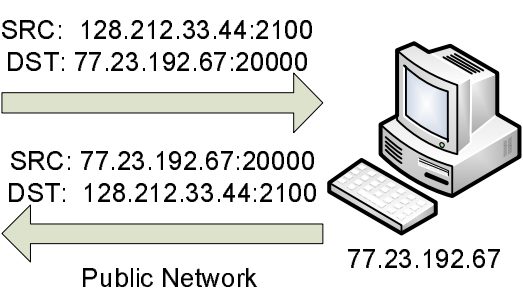

Fig. 2. A typical NAT interaction. The peer behind a NAT has a private address. When the packet is sent through the NAT, the NAT translates the source information into a public mapping, keeping the original source information so that if a packet from the remote peer comes back, it can be translated and delivered to the original source.

IP supports multicasting to groups interested in a common service. In the case of bootstrapping a P2P system [6], [15], all peers would be members of a specific group. When a new peer comes online, it queries the group for connection information and connects to those that respond. The approach, by itself, requires that all peers are located in a multicast capable network, restricting this approach typically to local area networks.

A large-scale structured overlay [17], [18] could enable peers to publish their information into a dedicated location for their service or application and then query that list to obtain a list of online peers. Peers could search for other peers in their overlay and connect with them using their connection information. Since the service would be a large-scale system, it could easily be bootstrapped by a dedicated bootstrap or host caches. As it stands, the described works were position papers and the systems have not been fully fleshed out. The primary challenge in relationship to small, ad-hoc networks is that it lacks details bootstrapping of peers behind NATs into overlays as it provides only a means for rendezvous and no reflection nor relaying.

\section{B. NAT Hampering the Bootstrap Process}

As of 2010, the majority of the Internet is connected via Internet Protocol (IP) version 4. This protocol has a quickly approached limit of addresses available, only $2^{32}$ (approximately 4 billion). With the Earth's population at over 6.8 billion and each individual potentially having multiple devices with Internet connectivity, the IPv4 limitation is becoming more and more apparent. Addressing this issue are two approaches: 1) the use of NATs to enable many machines and devices to share a single IP address but preventing bidirectional connection initiation, and 2) IPv6 which supports $2^{128}$ addresses. The use of NATs, as shown in Figure 2, complicates the bootstrapping of $\mathrm{P} 2 \mathrm{P}$ systems as it prevents peers from simply exchanging addresses with each other to form connections, as the addresses may not be public. In addition, firewalls may prevent peers from receiving incoming connections. Thus, while the eventual widespread use IPv6 may eliminate the need for address translation, it does not deal with the issue of firewalls preventing P2P applications from communicating, and it is not clear that IPv6 users will not continue to rely on NAT/firewall devices to provide a well-defined boundary of isolation for their local networks.
There are a handful of recognized NAT devices as presented in [19], [20]. The following list focuses on the more prevalent types:

- Full cone - All requests from the same internal IP and port are mapped to a static external IP and port, thus any external host can communicate with the internal host once a mapping has been made.

- Restricted cone - Like a full cone, but it requires that the internal host has sent a message to the external host before the NAT will pass the packets.

- Port restricted cone - Like a restricted cone, but it requires that the internal host has sent the packet to the external hosts specific port, before the NAT will pass packets.

- Symmetric - Each source and destination pair have no relation, thus only a machine receiving a message from an internal host can send a message back.

Two peers behind NATs will be unable to directly communicate with each other without assistance in acquiring routable addresses. Section III-A describes techniques that enable peers to acquire routable addresses, Section III-B describes relaying solutions, the alternative when two peers are unable to communicate directly, and Section III-C describes mechanisms for peers to identify each other to exchange address and relaying information.

\section{CORE REQUIREMENTS}

As presented in the preceding sections, a solution to bootstrapping small P2P overlays must address several challenges, namely reflection, rendezvous, and relaying. In this section, we present a generic solution to this problem. At the basis of our solution is the use of a publicly available free-to-join public overlay. In order to support these features the public overlay must have mechanisms for peers to obtain a public network identity (reflection); search for other peers that are bootstrapping the same P2P service (rendezvous); and send messages to peers through the overlay (relaying). These are the minimum requirements to bootstrap a decentralized, P2P system when all peers are behind NATs.

\section{A. Reflection}

Reflection provides a peer with a globally-addressable identifier, which can be shared with other peers so that it can receive incoming messages. Without reflection, peers on different networks with non-public addresses would not have 
routable addresses with which to communicate with each other. Reflection does not need to be limited to IP. For example, when a peer joins a service, such as a chat application or a $\mathrm{P} 2 \mathrm{P}$ system, the overlay provides a unique identifier, which also serves as a form of reflection.

In IP communication, reflection can enable NAT traversal. The simplest method for NAT traversal is the multiplexing of a single UDP socket, IP address and port combination. This behavior can be supported through either local configuration or remote assistance. The local configuration approach relies on the local router supporting either UPnP [21] or port forwarding / tracking. In many cases, UPnP is not enabled by default and in most commercial venues it will rarely be enabled. Port forwarding / tracking require a more detailed configuration of a router, outside the comfort range of many individuals and is not uniform across routers. A peer using UPnP needs no further services, as UPnP enables a peer to set and obtain both public IP address and port mappings. Port forwarding and tracking mechanisms still require that the user obtains and inputs into the application their public IP address or use in-band assistance described next.

In the remotely assisted scenario, a peer first sends a message to a reflection provider, perhaps using STUN [22]. The response from the provider tells the peer from which IP address and port the message was sent. In the case of all cone NATs, this will create a binding so that the peer can then share that IP address and port with other peers behind NATs. When the two peers communicate simultaneously, all types of cone NATs can be traversed; the timing of messages needs to be carefully considered, however, since NAT mappings may change over time. So long as one peer is behind a cone NAT, NAT traversal using this mechanism is possible. The situation becomes complicated when both peers are behind symmetric NATs, or when either one of them have a firewall prevent UDP communication.

Peers behind symmetric NATs cannot easily communicate with each other, since there is no relation between remote hosts and ports and local ports. Further complicating the matter is that there are various types of symmetric NATs, having behaviors similar to the various cone NAT types. In [23] the authors describe methods to traverse these NATs so long as there is a predictable pattern to port selection.

Unlike UDP, TCP NAT traversal is complicated by the state associated with TCP. In many systems, the socket API can be used to enable a peer to both listen for incoming connections and form outgoing connections using the same local addressing information. According to [24], this method works for various types of systems though the success rate on NATs is low, $40 \%$. Other mechanisms rely on out-ofband communication [25], or use of complicated predictive models [26].

\section{B. Relaying}

NAT traversal services only deal with one aspect of the bootstrap problem: reflection. That is, peers are able to obtain a public address for receiving incoming connections. They provide no means for users to exchange addresses with each other or perform simultaneous open to traverse restrictive NATs. To address this issue, many systems incorporate these NAT traversal libraries and use intermediaries to exchange addresses as a method of relaying. Another form of relaying exists when two peers are unable to form direct IP connections with each other.

The most common method for relaying in IP is the use of TURN [27], or Traversal Using Relay NAT. A peer using TURN obtains a public IP address and port that can be used as a forwarding address. When a remote peer sends to this address, the TURN server will forward the response to the peer who has been allocated that mapping. The lack of abstraction in TURN makes the system heavily centralized, making its application in small-scale systems complicated.

In overlays, peers typically have an abstracted identifier that does not associate them with a single server enabling more decentralized approaches to relaying. When a remote peer sends a message to the identifier, the overlay should translate the identifier into network level addresses and forward it to the destination.

Relaying must, also, have reliability features like UDP enabling peers to exchange various sized, arbitrary messages. When a peer sends a message, it should expect the remote peer should receive it in a reasonable amount of time or not at all. If the sending peer does not receive a response within a reasonable amount of time, follow up requests can be sent until successful or it is deemed the remote party is no longer online.

Finally, the service should be asynchronous or event driven. The previous requirements would allow peers to relay through a message board or even by posting messages to a DHT. The problem with these two approaches is that peers may very well communicate for long periods of time using these services. That means the potential for posting large amounts of data to a service that will retain it and constantly querying the service to determine if an update is available. Both of these are highly undesirable and may be viewed as denial of service or spam attacks.

\section{Rendezvous}

A rendezvous service should allow peers to discover peers interested in the same service and provide a global identifier to contact that peer. In the simplest case, a peer could randomly probe other peers on the Internet until it finds a matching peer. This approach is unreasonable if overlay is small and even more so if the peers are behind NATs, as the NATs may very well ignore the requests even if a peer behind the NAT is actively looking for that overlay as well.

Given an overlay, the most straightforward mechanism for rendezvousing is the use of a broadcast query to determine if any other peers are using the same service. In small enough overlays, this is a perfectly reasonable approach, though in large scale systems such as Gnutella, the approach is not scalable. 
Rather than attempt to make a single unified rendezvous technique, using a unique approach for each overlay can enable more efficient forms of rendezvous increasing the likelihood of peers to find a mutual peer and doing so more quickly. For example, in the case of a DHT, peers can use a single DHT key to store multiple values, all of which would be addresses used to communicate with peers in the overlay. Alternatively, in a system like BitTorrent, peers could use the same tracker and become "seeds" to the same virtual file.

\section{IMPLEMENTATIONS}

Table I reviews various overlays, the majority of which are high availability, public, free-to-join overlays, though some research only overlays are included. From this list, we chose to extend Brunet and XMPP to support private overlay bootstrapping. Brunet provides a structured $\mathrm{P} 2 \mathrm{P}$ infrastructure, though lacks an active, large-scale deployment outside of academic institutions due to being rooted in an academic project. XMPP, on the other hand, enables connections between friends with routing occurring across a distributed overlay.

Our implementation makes heavy use of the transports incorporated into Brunet [2]. The key distinguishing feature of this library is the abstraction of sending over a communication link as it supports primitives similar to "send" and "receive" that enables the ability to create $\mathrm{P} 2 \mathrm{P}$ communication channels over a variety of transports. In the next sections, we will describe how we extended Brunet to be self-bootstrapping as well as extensions to enable bootstrapping from XMPP.

Our application of structured overlays as the basis private overlays focuses on the autonomous, self-managing property of the overlay network rather than the ability to scale to very large numbers. This has also been the motivation of related work which has employed structured overlays in systems in the order of 10s to 100s of nodes. For example, Amazon's shopping cart runs on Dynamo [31] using a "couple of hundred of nodes" or less. Facebook provides an inbox search system using Cassandra [32] running on "600+ cores". Structured overlays simplify organization of an overlay and provide each member a unique identifier abstracted from the underlying network. As mentioned in the cited works, they provide high availability and autonomic features that can handle churn well. When used in small networks, most structured overlays (including Brunet and Pastry) in effect act as $O(1)$ systems, selforganizing links that establish all-to-all connectivity among peers. Brunet explicitly supports all-to-all connectivity, though in some cases may require constrained peers to route through relays. This can further be ensured by setting the amount of near connections for the infrastructures, which in Brunet is configurable at run time.

The XMPP library we used is called Jabber-Net. Each connection between peers is uniquely identified by employing socket like concepts, i.e., a pair of addresses and ports. The basic representation for this constitutes a pair of identifiers of the form "brunet://P2P_ID:PORT", where each peer has a unique ID and port associated for the local and remote entity. The XMPP implementation has a similar for- mat: “xmpp://USERNAME@DOMAIN:PORT/RESOURCE”, again one identifier for the local peer and one for the remote.

\section{A. Bootstrapping Private Overlays Using Brunet}

Prior to our work, Brunet bootstrapped using a recently online cache of peers and IP multicast. We have implemented Brunet to support STUN, such that, with every connection Brunet makes, peers inform each other of their view of the remote peers network state, a form of passive reflection. Peers also generate a unique 160-bit node identifier that can be used in the overlay as a directly receive packets regardless of the underlay conditions.

In a single overlay, Brunet supports relaying either through the overlay or pseudo direct connections called "Tunnels" [30], where peers route to each other through common neighboring connections. The relaying in this context is used either to maintain a necessary overlay connection, or to exchange intentions to connect with each other through "ConnectToMe" messages. Thus when a peer desires a connection to another, both peers simultaneously attempt to connect to each other after exchanging endpoints discovered through reflection using the overlay relay mechanisms, dealing with the issue of more restrictive cone NATs and the case when the peer is behind a non-traversable NAT.

To support relaying within the scope of a private overlay, we further extended Brunet's transport library to support treating an existing overlay as a medium for point-to-point communication. This is called a "Subring" transport, because it supports the abstraction of multiple private sub-rings within a common large structured ring. When the private overlay transmits data across the public overlay, the private overlay packet is encapsulated (and possibly encrypted) in a packet that ensures it will be delivered to the correct private destination, and then encapsulated into a greedy routing packet. At which point, the packet is sent to the remote peer through the public overlay. In order to instruct peers to establish "Subring" links, they exchange an identifier of the form "brunet://P2P_ID".

Peers store their "Subring" identifiers into the DHT for rendezvous. The DHT provides a scalable and self-maintaining mechanism for maintaining a bootstrap, so long as the DHT supports multiple values at the same key, as Brunet does. The key used for the DHT rendezvous is a hash of the services name and its version number, which we call a namespace. Peers can then query this entry in the DHT to obtain a list of peers in the private overlay. Since DHTs are soft-state, or lease systems, where data is released after a certain period of time, an online peer must actively maintain its DHT entry. In the case that a peer goes offline, the DHT will automatically remove the value after its lease has expired.

The final challenge faced was the application of Brunet's reflection service for the private overlay. There were two directions we could have gone. The first would have been to extend Brunet to support STUN in each of the remote servers and then have a private node query them for their public information. The problem with this approach is that it would require maintaining additional state in order to discern 


\begin{tabular}{|c|c|c|c|c|}
\hline & Description & Reflection & Rendezvous & Relay \\
\hline BitTorrent & $\begin{array}{l}\text { Default BitTorrent implementations rely on a } \\
\text { centralized tracker to provide the initial boot- } \\
\text { strapping. Peers can establish new connections } \\
\text { through information obtained from established } \\
\text { connections. This relegates the tracker as a } \\
\text { means of monitoring the state of the file dis- } \\
\text { tribution. BitTorrent specifies a protocol, though } \\
\text { each client may support additional features not } \\
\text { covered by the protocol. }\end{array}$ & $\begin{array}{l}\text { The current specification } \\
\text { does not support NAT } \\
\text { traversal, though future } \\
\text { versions may potentially } \\
\text { use UDP NAT traversal. } \\
\text { At which point, BitTorrent } \\
\text { may support a reflection } \\
\text { service. }\end{array}$ & $\begin{array}{l}\text { Peers can register as seeds } \\
\text { to the same file hash, thus } \\
\text { their IP address will be } \\
\text { stored with the tracker. }\end{array}$ & $\begin{array}{l}\text { Peers receive each other's } \\
\text { IP addresses from the } \\
\text { tracker, there is no inher- } \\
\text { ent relaying. }\end{array}$ \\
\hline XMPP & $\begin{array}{l}\text { XMPP consists of a federation of distributed } \\
\text { servers. Peers must register an account with a } \\
\text { server, though registration can be done through } \\
\text { XMPP APIs without user interaction. XMPP is } \\
\text { not a traditional P2P system, though it has some } \\
\text { P2P features. XMPP servers on distinct servers } \\
\text { are able to communicate with each other. Links } \\
\text { between servers are created based upon client } \\
\text { demand. During link creation, servers exchange } \\
\text { XMPP Federation signed certificates. }\end{array}$ & $\begin{array}{l}\text { While not provided by all } \\
\text { XMPP servers, there exist } \\
\text { extensions for NAT traver- } \\
\text { sal. GoogleTalk, for exam- } \\
\text { ple, provides both STUN } \\
\text { and TURN servers. }\end{array}$ & $\begin{array}{l}\text { Similar to Skype, XMPP } \\
\text { friends can broadcast } \\
\text { queries to each other to } \\
\text { find other peers using } \\
\text { the same P2P service. } \\
\text { Thus XMPP supports } \\
\text { rendezvous. }\end{array}$ & $\begin{array}{l}\text { The XMPP specification } \\
\text { allows peers to exchange } \\
\text { arbitrary out-of-band } \\
\text { communication with } \\
\text { each other. Most servers } \\
\text { support this behavior, } \\
\text { even when sent across the } \\
\text { Federation. Thus XMPP } \\
\text { supports relaying. }\end{array}$ \\
\hline Kademlia [9] & $\begin{array}{l}\text { There exists two popular Kademlia systems, one } \\
\text { used by many BitTorrent systems, Kad, and the } \\
\text { other used by Gnutella, called Mojito. Kadem- } \\
\text { lia implements an iterative structured overlays, } \\
\text { where peers query each other directly when } \\
\text { searching the overlay. Thus all resources of a } \\
\text { Kademlia overlay must have a publicly address- } \\
\text { able network endpoint. }\end{array}$ & $\begin{array}{l}\text { Existing implementations } \\
\text { of Kademlia do not sup- } \\
\text { port mechanims for peers } \\
\text { to determine their network } \\
\text { identity. }\end{array}$ & $\begin{array}{l}\text { Peers can use the DHT } \\
\text { as a rendezvous service, } \\
\text { storing their connectiv- } \\
\text { ity information in the } \\
\text { DHT at key location: } \\
\text { hash }(S E R V I C E) \text {. }\end{array}$ & $\begin{array}{l}\text { An iterative structured } \\
\text { overlay has no support } \\
\text { for relaying messages. }\end{array}$ \\
\hline OpenDHT [28] & $\begin{array}{l}\text { OpenDHT is a recently decommissioned DHT } \\
\text { running on PlanetLab. OpenDHT is built using } \\
\text { Bamboo, a Pastry-like protocol [6]. Pastry im- } \\
\text { plements recursive routing, peers route messages } \\
\text { through the overlay. }\end{array}$ & $\begin{array}{l}\text { Existing implementations } \\
\text { of Bamboo and Pastry } \\
\text { do not support mechan- } \\
\text { ims for peers to deter- } \\
\text { mine their network iden- } \\
\text { tity. Though this is ongo- } \\
\text { ing work. }\end{array}$ & $\begin{array}{l}\text { Peers can use the DHT } \\
\text { as a rendezvous service, } \\
\text { storing their connectiv- } \\
\text { ity information in the } \\
\text { DHT at key location: } \\
\text { hash }(S E R V I C E) \text {. }\end{array}$ & $\begin{array}{l}\text { Because Pastry uses re- } \\
\text { cursive routing, it can be } \\
\text { used as a relay. Further- } \\
\text { more, extensions to Pastry } \\
\text { have enabled explicit re- } \\
\text { lays called virtual connec- } \\
\text { tions [29]. }\end{array}$ \\
\hline Brunet [2] & $\begin{array}{l}\text { Brunet like OpenDHT is a freely available DHT } \\
\text { running on PlanetLab, though still in active } \\
\text { development. Brunet creates a Symphony [8] } \\
\text { overlay using recursive routing. }\end{array}$ & $\begin{array}{l}\text { Brunet supports inherent } \\
\text { reflection services, when a } \\
\text { peer forms a connection } \\
\text { with a remote peer, the } \\
\text { peers exchange their view } \\
\text { of each other. }\end{array}$ & $\begin{array}{l}\text { Peers can use the DHT } \\
\text { as a rendezvous service, } \\
\text { storing their connectiv- } \\
\text { ity information in the } \\
\text { DHT at key location: } \\
\text { hash }(S E R V I C E) \text {. }\end{array}$ & $\begin{array}{l}\text { Like Pastry, Brunet sup- } \\
\text { ports recursive routing and } \\
\text { relays called tunnels [30]. }\end{array}$ \\
\hline
\end{tabular}

TABLE I

Public AND RESEARCH Overlays

which of the remote peers are on public addresses and can provide STUN services.

Instead, we opted to multiplex the socket used for the public overlay as it already had gone through the process of "reflection". We call the multiplexing of a single socket for multiple overlay "Pathing". In this context, the public and private overlays are given a virtual transport layer that hooks into an underlying transport layer, thus not limited purely to socket transport layers. When peers exchange identifiers, instead of transmitting a simple identifier like "udp://192.168.1.1:15222", the "Pathing" library extends it to "udp://192.168.1.1:15222/path", where each path will signify a unique overlay.

Our completed approach is illustrated in Figure 3. The approach of "Subring" and "Pathing" enabled the reuse of the core components of Brunet. Using "Subring" enables peers to form bootstrap connections to then exchange "ConnectToMe" messages. If the direct connections failed, then the "Subring" connections could be used as permanent connections. The use of "Pathing" meant reuse of existing NAT traversal techniques 


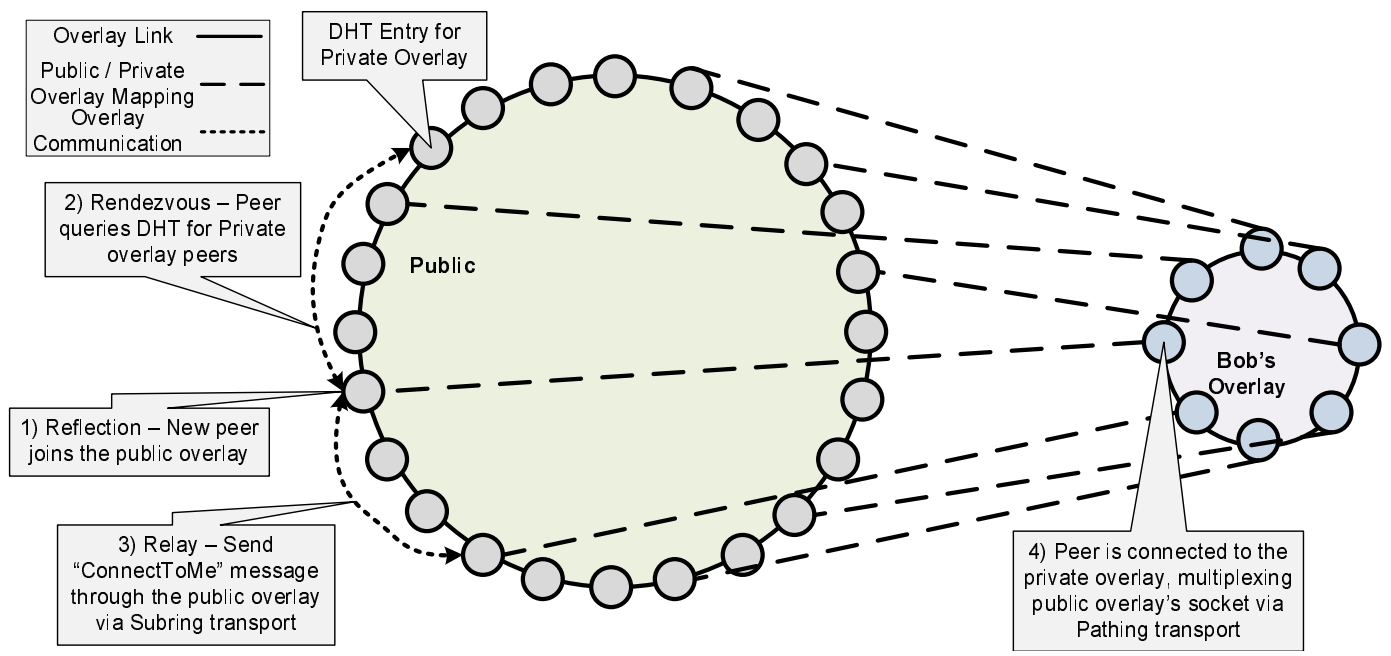

Fig. 3. Bootstrapping a P2P system using Brunet.

and limited the amount of system resources required to run multiple overlays. In terms of total lines of code, these abstractions enabled a recursive overlay bootstrapping with a relatively small code footprint — less than 1000 lines of code.

\section{B. Bootstrapping Private Overlays Using XMPP}

In addition to supporting recursive bootstrapping of private overlays, the techniques described above can be extended to use a different public overlay - an XMPP-based federation - to support the bootstrapping of private overlays. The key features that make XMPP attractive are the distributed nature of the federation and the openness of the protocol. As of December 2009, there are over 70 active XMPP servers in the XMPP Federation [33]. These include GoogleTalk, Jabber.org, and Live Journal Talk.

In XMPP, each user has a unique identifier of the form "username@domain". Where the domain specifies the client XMPP server and the username explicitly identifies a single individual. XMPP supports concurrent instances for each user by appending a resource identifier to the user ID: "username@domain/resource". A resource identifier can either be provided by the client or generated by the server. For users in the same domain, the server forwards the message from source to destination. When two users are in different domains, the sender's server forwards the message to the receiver's server, who then relays it to the receiver. Peers are able to send text messages to each other as well as arbitrary binary messages called "IQ".

Peer relationships are maintained by the server. Peer initiate them through an in-band subscription mechanism based upon "IQ", allowing clients to handle the process of adding and removing peers. Once peers have established a connection or subscription, they are informed through a "Presence" notification that the peer has come online, this include the full user identifier.

The first form of reflection in XMPP is the unique client identifier. Another is an IP reflection service available from some XMPP service providers called "Jingle" [34]. "Jingle" uses "IQ" to determine available STUN and TURN servers.
Fortunately, these services are provided free of charge through GoogleTalk. In Brunet, we extended the UDP transport to support querying STUN servers so that it can obtain an address mapping and keep it open. STUNs protocol sets the first two bits to 0 in all messages, thus we used that and the STUN cookie to distinguish it from other messages.

In order to support the situation where two peers are unable to communicate through the exchanged addresses, we have extended XMPP "IQ" as a transport to support relaying. Once a peer has formed a connection through XMPP, they are then able to attempt simultaneous connection attempts, in the same fashion as the "Subring" transport, further increasing the likelihood of a connection. If that does not succeed, the peers can still relay through XMPP. This approach also has the benefit that, if a XMPP server does not support "Jingle", the two peers can still form links with each other. Since Brunet internally supports IP reflection, eventually, if one of the peers in the system has a public address, it will automatically assist the other peers into forming direct links with each other.

Rendezvous uses a two step approach. First peers advertise their use of private overlay in the resource identifier. The name is hashed to ensure that the users complete identifier does not extend past 1,023 bytes, the maximum length for these identifiers. In addition, a cryptographically generated random number is appended to the resource identifier to distinguish between multiple instances of the users application in the same private overlay. Once a peer receives a presence notification from a remote peer and the base components match, that is the hash of the service, the peer adds it to a list of known online peers. If the peer lacks connections, the system broadcasts to that list a request for addresses. The peers respond with a list of addresses including UDP, TCP, and XMPP addresses, concluding rendezvous.

Ideally, peers would not need to create XMPP connections with each other; if they are on a public address, the rendezvous phase alone will suffice. In the case that they are not on public addresses, peers can first obtain their public address through STUN, then form an XMPP connection with each other, and finally perform simultaneous connection attempts. 
This full text paper was peer reviewed at the direction of IEEE Communications Society subject matter experts for publication in the IEEE P2P 2010 proceedings.

If NAT traversal fails, the peers can continue routing through the XMPP connection. Due to the abstractions employed by the transport library, the additional support for XMPP-based bootstrapping required only an additional 700 lines of code to Brunet and no modification to the core system.

\section{Evaluating OVERlay Bootstrapping}

In this section, we present a qualitative evaluation of our prototype bootstrapping a small-scale network and also share some experiences with deploying overlays.

\section{A. Deployment Experiments}

The purpose of our experiment is to verify that our techniques work and what overheads should be expected in using Brunet and XMPP to bootstrap an overlay. Rather than an extensive experiment overly focused on overheads of Brunet and XMPP, this experiment is primarily focused on the feasibility of forming small-scale overlays among network-constrained peers. The experiment represents 5 peers desiring all-to-all direct connectivity, a feature transparently available to them if they bootstrap into a private Brunet overlay. The experiments were run on peers deployed on 5 distinct virtual machines each virtual machine had its own separate NAT, and thus peers were unable to communicate directly without assistance.

The public Brunet overlay used in this experiment consisted of over 600 nodes and ran on PlanetLab. PlanetLab [35] is a consortium of research institutes sharing hundreds of globally distributed network and computing resources. GoogleTalk provided the XMPP overlay used in this experiment. Though this experiment does not take into advantage the features of the XMPP Federation, this aspect is presented in more detail in the next section reviewing experiences deploying overlays using XMPP.

In the experiment, 5 P2P nodes were started simultaneously, while measuring the time spent for reflection, rendezvous, reflection, and connection. The results are presented in Table II. For XMPP, these are translated as follows: reflection measures the time to obtain IP addresses from the STUN server, rendezvous is the time to receive a presence notification, relaying is the time to receive a message across XMPP, and connected is once all nodes in the private overlay has all-to-all connectivity. For Brunet, these are translated as follows: reflection measures the time to connect to the public overlay, rendezvous is the time to query the DHT, relaying is the average time to send a message across the overlay, and connected is the time until the private overlay has all-to-all connectivity. The results are highly correlated to timeouts in Brunet, which employs a mixture of events and polling to stabilize the overlay, as well as the latency between the client and GoogleTalk. As this was more of a qualitative experiment, the results are clear: private overlays providing all-to-all connectivity among NATed nodes can bootstrap within a very reasonable amount of time.

\section{B. Deployment Experiences}

Recently, Facebook announced that they would be supporting XMPP as a means to connect into Facebook chat. This was rather exciting and further motivated this work, as

\begin{tabular}{|c||c|c|c|c|}
\hline & Reflection & Rendezvous & Relaying & Connected \\
\hline \hline XMPP & .035 & .110 & .243 & 20.3 \\
\hline Brunet & 3.05 & .330 & .533 & 23.22 \\
\hline \multicolumn{4}{|c}{ TABLE II } \\
\hline
\end{tabular}

TIME IN SECONDS FOR VARIOUS PRIVATE OVERLAY OPERATIONS

Facebook has over 400 million active users, which would have made their XMPP overlay, potentially, the largest freeto-join overlay. Unfortunately, Facebook does not employ a traditional XMPP setup, instead it provides a proxy into their chat network, preventing features like arbitrary IQs and other forms of out-of-band messages to be exchanged between peers. User identifiers are also translated, so a peer cannot obtain a remote peers real identifier. Thus there exists no out-ofband mechanism for rendezvous. Peers could potentially send rendezvous messages through the in-band XMPP messaging, but this may be viewed by most recipients as spam as it would arrive as normal chat messages. The lesson learned was that XMPP servers not associated with the Federation will not necessarily support features necessary to bootstrap.

During initial tests in verifying the workings of the XMPP code base, we bootstrapped a private Brunet overlay on PlanetLab through various XMPP service providers. We discovered that some servers were ignoring clients on PlanetLab. Another server crashed after 257 concurrent instances of the same account logged in. Unfortunately, the provider had no contact information available, so we were unable to determine if our test caused the crash. Though there did exist some servers that had no trouble hosting over 600 concurrent instances running on PlanetLab.

Once the system was running on PlanetLab, more tests were performed to determine the ability to bootstrap across the XMPP Federation. We formed friendships, or subscriptions, between users across a few different XMPP service providers. In the most evaluated case, a single peer on GoogleTalk along with 600 peers on PlanetLab system using jabber.rootbash.com, the GoogleTalk peer would not always receive presence notifications for all peers online, though always would receive some. When a peer began the relaying mechanism, it would broadcast to every peer from whom it received a presence notification. When performing this between GoogleTalk and rootbash, the GoogleTalk peer would not receive a response. Though in reducing the broadcast to a random selection of 10 peers, every 10 seconds until the GoogleTalk peer was connected, the peer received responses. The behavior indicates that the XMPP servers may have been filtering to prevent denial of service attacks.

Peers on the same XMPP server seem to be connected very quickly, though peers on different services can take significantly longer. For example, when bootstrapping a single peer from GoogleTalk into the rootbash system, it always took 1 minute for the node to become fully connected to the private overlay. When the peer used rootbash, the peer always connected within 30 seconds. It seems as if the communication between XMPP servers was being delayed for some reason. The same behavior was not experienced, when chatting between the two peers. 


\section{CONCLUSION}

In this paper,we have established the requirements for bootstrapping small-scale P2P overlays: reflection, relaying, and rendezvous. Reflection is required so that peers behind NATs and firewalls can obtain public addresses to share with remote peers. Relaying provides a means for peers to coordinate simultaneous connection attempts and a fall-back in the case that NAT traversal attempts fail. Rendezvous is a common problem, even for large-scale systems; peers must have a mechanism to find other peers connecting to are in the same overlay.

We extended Brunet to support bootstrapping small-scale overlays from another Brunet overlay as well as XMPP. $\mathrm{XMPP}$ is an ideal choice as it already supports the key features required for bootstrapping small-scale P2P overlays. The Brunet solution offers interesting insights. In particular, we have found that the ability to multiplex a single socket or transport for multiple overlays enables overlays that use UDP as a transport with NAT traversal support to leverage NAT traversal in one domains in all domains, i.e., the peer does not need to maintain unique NAT mappings for each overlay. XMPP provides a reliable, production ready, mechanism of bootstrapping overlays as the service is hosted by a distributed set of providers, each offering interoperability. Our prototype clearly identifies methods for reflection, relaying, and rendezvous and can assist in discovering methods for doing so in similar systems.

For future work, we plan on investigating how peers can leverage existing DHT deployments, such as Kad or Mojito, for rendezvous, form friendships automatically in XMPP, and continue the bootstrap process using XMPP framework discussed in this paper. Using this approach would enable the XMPP solution to be extended to bootstrap overlays that are not based on social connections alone.

\section{ACKNOWLEDGMENT}

We thank the anonymous reviewers for their useful comments and feedback. This work is sponsored by the National Science Foundation under awards 0751112 and 0721867. Any opinions, findings and conclusions or recommendations expressed in this material are those of the authors and do not necessarily reflect the views of the NSF.

\section{REFERENCES}

[1] P. Saint-Andre, "RFC 3920 extensible messaging and presence protocol (XMPP): Core," October 2004.

[2] P. O. Boykin, et al., "A symphony conducted by brunet," http://arxiv. org/abs/0709.4048, 2007.

[3] P. S. Juste, D. Wolinsky, P. O. Boykin, M. J. Covington, and R. J. Figueiredo, "Socialvpn: Enabling wide-area collaboration with integrated social and overlay networks," 2010.

[4] T. Klingberg and R. Manfredi, "Gnutella 0.6," http://rfc-gnutella. sourceforge.net/src/rfc-0_6-draft.html, June 2002.

[5] hex, "The fasttrack protocol," http://cvs.berlios.de/cgi-bin/viewcvs.cgi/ gift-fasttrack/giFT-FastTrack\%/PROTOCOL, September 2004.

[6] A. Rowstron and P. Druschel, "Pastry: Scalable, decentralized object location and routing for large-scale peer-to-peer systems," in International Conference on Distributed Systems Platforms (Middleware), November 2001.
[7] I. Stoica, et al., "Chord: A scalable Peer-To-Peer lookup service for internet applications," in SIGCOMM, 2001.

[8] G. S. Manku, M. Bawa, and P. Raghavan, "Symphony: distributed hashing in a small world," in USITS, 2003.

[9] P. Maymounkov and D. Mazières, "Kademlia: A peer-to-peer information system based on the XOR metric," in IPTPS '02, 2002.

[10] S. Ratnasamy, P. Francis, S. Shenker, and M. Handley, "A scalable content-addressable network," in SIGCOMM, 2001.

[11] V. Ramasubramanian and E. G. Sirer, "Beehive: O(1)lookup performance for power-law query distributions in peer-to-peer overlays," in Symposium on Networked Systems Design and Implementation, 2004.

[12] H. Damfpling. (2003) Gnutella web caching system. http://www. gnucleus.com/gwebcache/specs.html.

[13] W. Ginolas. (2009) P2PVPN. http://p2pvpn.org.

[14] C. GauthierDickey and C. Grothoff, "Bootstrapping of peer-to-peer networks," 2008.

[15] C. Cramer, K. Kutzner, and T. Fuhrmann, "Bootstrapping locality-aware p2p networks," in in: The IEEE International Conference on Networks (ICON), 2004

[16] M. Knoll, A. Wacker, G. Schiele, and T. Weis, "Bootstrapping in peerto-peer systems," 2008.

[17] M. Castro, P. Druschel, A.-M. Kermarrec, and A. Rowstron, "One ring to rule them all: Service discover and binding in structured peer-to-peer overlay networks," in SIGOPS European Workshop, Sep. 2002.

[18] M. Conrad and H.-J. Hof, "A generic, self-organizing, and distributed bootstrap service for peer-to-peer networks," in International Workshop on Self-Organizing Systems (IWSOS), 2007.

[19] J. Rosenberg, J. Weinberger, C. Huitema, and R. Mahy. (2003) Stun - simple traversal of user datagram protocol (udp) through network address translators (nats).

[20] P. Srisuresh, B. Ford, and D. Kegel, RFC 5128 State of Peer-toPeer (P2P) Communication across Network Address Translators (NATs), March 2008.

[21] "UPnP device architecture 1.1," http://www.upnp.org/specs/arch/ UPnP-arch-DeviceArchitecture-v1.1.pdf, October 2008.

[22] J. Rosenberg, R. Mahy, P. Matthews, and D. Wing, "RFC 3489 session traversal utilities for nat (STUN)," October 2008.

[23] J. Rosenberg, "Interactive connectivity establishment (ICE): A protocol for network address translator (NAT) traversal for offer/answer protocols," http://tools.ietf.org/html/draft-ietf-mmusic-ice-19, October 2008.

[24] S. Perreault and J. Rosenberg, "TCP candidates with interactive connectivity establishment (ICE)," http://tools.ietf.org/html/ draft-ietf-mmusic-ice-tcp-08, October 2009.

[25] A. Rezmerita, T. Morlier, V. Neri, and F. Cappello, "Private virtual cluster: Infrastructure and protocol for instant grids," in Euro-Par, November 2006.

[26] A. Biggadike, D. Ferullo, G. Wilson, and A. Perrig, "NATBLASTER: Establishing TCP connections between hosts behind NATs," in ACM SIGCOMM Asia Workshop, April 2005.

[27] J. Rosenberg, R. Mahy, and P. Matthews. (2009) "traversal using relays around nat (turn)". http://tools.ietf.org/html/draft-ietf-behave-turn-16.

[28] S. Rhea, et al., "Opendht: a public dht service and its uses," in SIGCOMM, 2005

[29] A. Mislove, A. Post, A. Haeberlen, and P. Druschel, "Experiences in building and operating epost, a reliable peer-to-peer application," in EuroSys '06: Proceedings of the 1st ACM SIGOPS/EuroSys European Conference on Computer Systems 2006, 2006.

[30] A. Ganguly, et al., "Improving peer connectivity in wide-area overlays of virtual workstations," in HPDC, 2008.

[31] G. DeCandia, et al., "Dynamo: amazon's highly available key-value store," in SOSP '07: Proceedings of twenty-first ACM SIGOPS symposium on Operating systems principles. New York, NY, USA: ACM, 2007, pp. 205-220.

[32] A. Lakshman, "Cassandra a structured storage system on a P2P network," http://www.facebook.com/note.php?note_id=24413138919, August 2008.

[33] "Public XMPP services," http://xmpp.org/services/, December 2009.

[34] S. Ludwig, et al., "XEP-0166: Jingle," December 2009.

[35] B. Chun, D. Culler, T. Roscoe, A. Bavier, L. Peterson, M. Wawrzoniak, and M. Bowman, "Planetlab: an overlay testbed for broad-coverage services," SIGCOMM, 2003. 